\title{
Hypertension and Diabetes in Obesity
}

\author{
Kazuko Masuo, ${ }^{1,2}$ Michael L. Tuck, ${ }^{3}$ and Gavin W. Lambert ${ }^{1,4}$ \\ ${ }^{1}$ Human Neurotransmitters Laboratory, Baker IDI Heart \& Diabetes Institute, Melbourne, VIC 8008, Australia \\ ${ }^{2}$ Nucleus Network Ltd., Baker IDI Heart \& Diabetes Institute, Melbourne, VIC 8008, Australia \\ ${ }^{3}$ Endocrinology and Metabolic Disease, VA Greater Los Angeles Healthcare System and David Geffen School of Medicine at UCLA, \\ Los Angeles, CA 90073, USA \\ ${ }^{4}$ Faculty of Medicine, Nursing and Health Sciences, Monash University, Melbourne, VIC 3800, Australia
}

Correspondence should be addressed to Kazuko Masuo, kazuko.masuo@bakeridi.edu.au

Received 30 March 2011; Accepted 30 March 2011

Copyright (c) 2011 Kazuko Masuo et al. This is an open access article distributed under the Creative Commons Attribution License, which permits unrestricted use, distribution, and reproduction in any medium, provided the original work is properly cited.

The epidemic-like rise in the prevalence of obesity constitutes an undoubted and serious global health problem. Importantly, hypertension and diabetes are frequently associated with obesity and, together, constitute a significant burden in terms of patients' morbidity and escalating health care costs. When considered in isolation, obesity, hypertension, and diabetes are all associated with increased risk of the development of cardiovascular and renal complications; however, the coexistence of this triumvirate generates a substantial elevation in disease risk. The driving forces linking obesity, hypertension, and diabetes remain to be clarified due, in part, to the complex and multifactorial nature of the conditions that involve combinations of environmental, genetic, life style, and behavioural confounders. Additionally, it is recognized that neuroendocrine mechanisms, including insulin resistance, sympathetic nervous activation, and stimulation of the renin-angiotensin-aldosterone system (RAAS), are also involved [1-4].

This special issue on hypertension and type 2 diabetes related to obesity includes several epidemiological studies focusing on the prevalence of metabolic syndrome, type 2 diabetes, and hypertension. New data emanating from Peru, Ethiopia, Sudan, Egypt, and Nepal documents the prevalence of cardiometabolic disease in these countries as being similar to that reported in westernized countries such as the USA, Canada, Australia, and European countries as well as Japan $[5,6]$. Importantly, an emerging body of data, such as that presented by Professor C. Brufani et al., highlights the importance of obesity in children. They reported the contribution of birth weight to central fat depot and insulin sensitivity in metabolic syndrome in obese
Italian children. The challenge will be in designing and implementing effective strategies to arrest and reverse this pattern.

The strong linkage between hypertension and type 2 diabetes was reviewed by Professor E. Dean which provides insight into the mechanisms involved. Dr. S. Horita et al. reviewed the contribution of the kidneys, especially renal sodium transport, to the development of insulin resistance and hypertension in obesity. Diabetic patients and obese individuals frequently present with different circadian patterns of blood pressure compared to nondiabetic or nonobese subjects. A nondipping pattern is very common in obese hypertensive patients. In diabetic patients, ambulatory blood pressure monitoring provides a more robust measure in predicting future cardiovascular events than clinic blood pressure. Dr. C. Anigbogu et al. provided evidence that in rats the circadian rhythm of blood pressure and heart rate changes with progression of diabetes. Professor K. Eguchi reviewed recent epidemiological studies in diabetes and obesity using ambulatory blood pressure monitoring. Taken together, these observations demonstrate the importance of ambulatory blood pressure monitoring.

The first line of therapy for the treatment of type 2 diabetes and obesity-related hypertension is weight loss with lifestyle modifications such as diet and exercise. Nonpharmacological treatments were outlined by Dr. J. Pappachan et al. Another article by S. Guy et al. indicated that video gaming provided some benefit in initiating lifestyle modifications that aided in weight loss. Patients with diabetes and hypertension frequently present with atherogenic diseases and dyslipidemia. Dr. E. Morales-Villegas and colleagues 
provided a review indicating that statins are very effective in treating dyslipidemia and reducing overall cardiovascular risk.

This special issue covered a wide range of materials with a focus on type 2 diabetes and hypertension. Articles included epidemiology, physiology, and treatments. In summary, this issue demonstrated that (i) abdominal obesity is related to the high prevalence of hypertension and type 2 diabetes regardless of ethnicity, (ii) insulin resistance is a major mechanism linking the onset and development of hypertension and type 2 diabetes, and (iii) weight loss with diet and exercise is an important aspect in treating hypertension in type 2 diabetes and aids in increasing the efficacy of antihypertensive medications. Further investigations on mechanisms and genetics are needed in order to develop appropriate and effective therapeutic regimens in order to prevent and limit obesity-related illnesses such as hypertension and type 2 diabetes. Early intervention is vital, given emerging evidence of end-organ dysfunction in young overweight or obese individuals $[7,8]$.

\section{Acknowledgments}

The editors thank the authors of all submissions and the tireless reviewers for their critical assistance. The editors enjoyed the variety of articles and hope that this special issue is useful for clinical practice and research and hope that the content of this special issue facilitates the reduction of the global burden of obesity and obesity-related illness.

\author{
Kazuko Masuo \\ Michael L. Tuck \\ Gavin W. Lambert
}

[7] E. Lambert, C. I. Sari, T. Dawood et al., "Sympathetic nervous system activity is associated with obesity-induced subclinical organ damage in young adults," Hypertension, vol. 56, no. 3, pp. 351-358, 2010.

[8] K. Masuo, G. W. Lambert, M. D. Esler, H. Rakugi, T. Ogihara, and M. P. Schlaich, "The role of sympathetic nervous activity in renal injury and end-stage renal disease," Hypertension Research, vol. 33, no. 6, pp. 521-528, 2010.

\section{References}

[1] K. Masuo, "Role of beta2- and beta33 adrenoceptor polymorphisms in hypertension and metabolic syndrome," International Journal of Hypertension, vol. 2010, Article ID 832821, 2010.

[2] M. D. Esler, N. E. Straznicky, N. Eikelis, K. Masuo, G. Lambert, and E. A. Lambert, "Mechanisms of sympathetic activation in obesity-related hypertension," Hypertension, vol. 48, pp. 787796, 2008.

[3] M. Esler, G. Jennings, and G. Lambert, "Noradrenaline release and the pathophysiology of primary human hypertension," American Journal of Hypertension, vol. 2, no. 3, pp. 1405-1465, 1989.

[4] K. Masuo, "Obesity-related hypertension: role of the sympathetic nervous system, insulin, and leptin," Current Hypertension Reports, vol. 4, no. 2, pp. 112-118, 2002.

[5] A. H. Mokdad, E. S. Ford, B. A. Bowman et al., "Prevalence of obesity, diabetes, and obesity-related health risk factors, 2001," Journal of the American Medical Association, vol. 289, no. 1, pp. 76-79, 2003.

[6] E. L. M. Barr, A. J. Cameron, B. Balkau et al., "HOMA insulin sensitivity index and the risk of all-cause mortality and cardiovascular disease events in the general population: the australian diabetes, obesity and lifestyle study (AusDiab) study," Diabetologia, vol. 53, no. 1, pp. 79-88, 2010. 


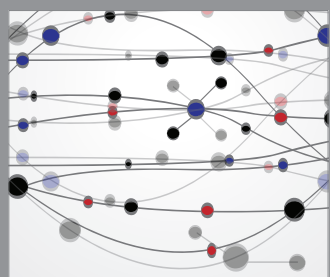

The Scientific World Journal
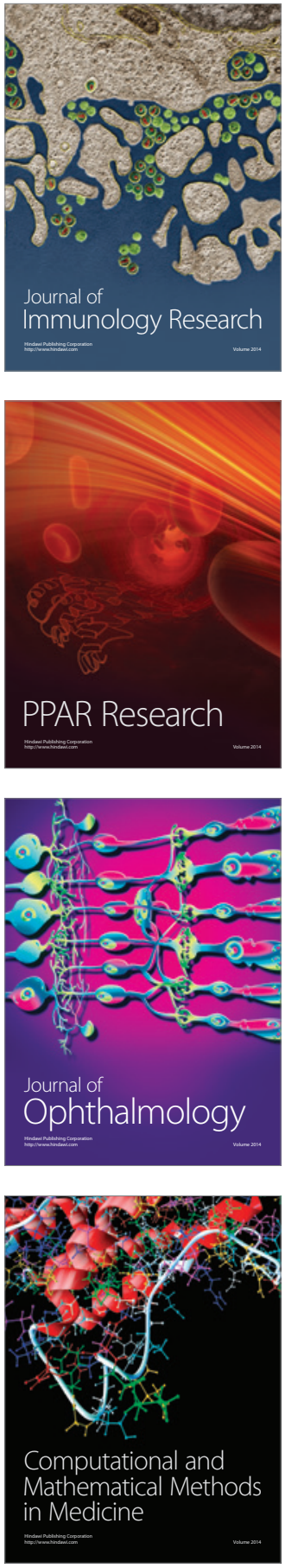

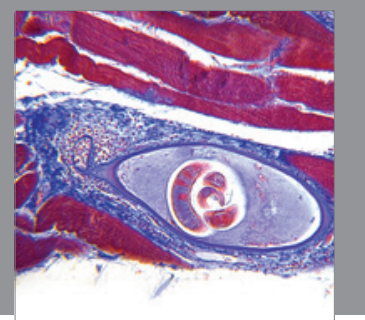

Gastroenterology

Research and Practice
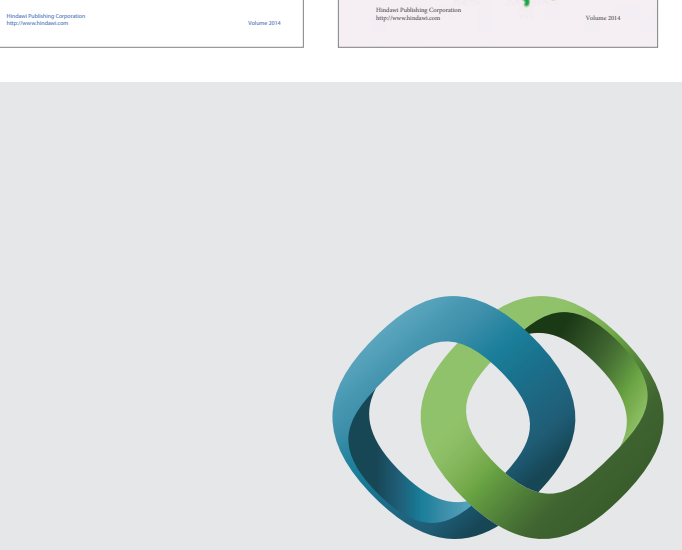

\section{Hindawi}

Submit your manuscripts at

http://www.hindawi.com
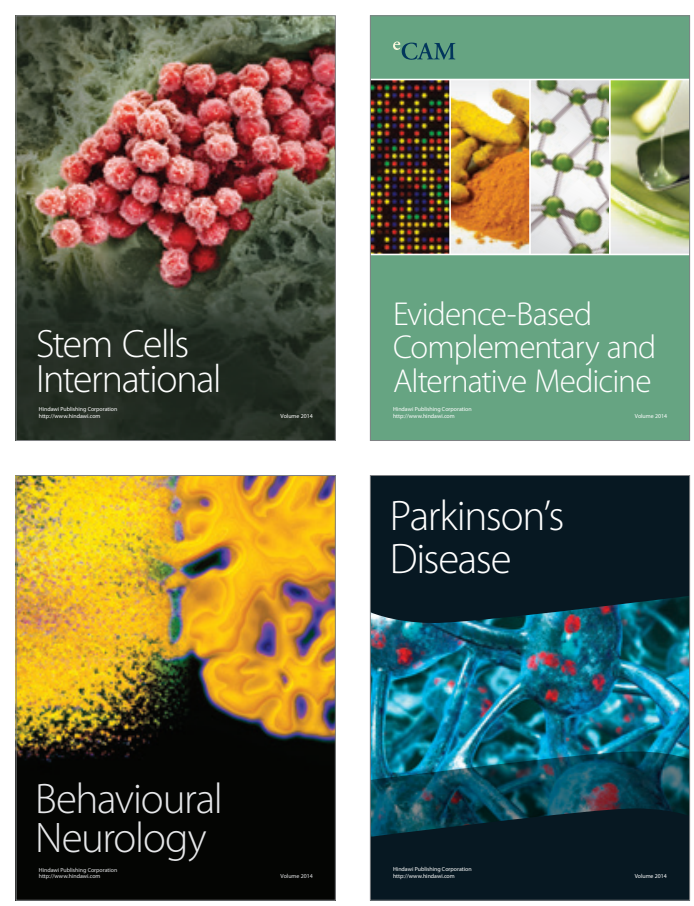

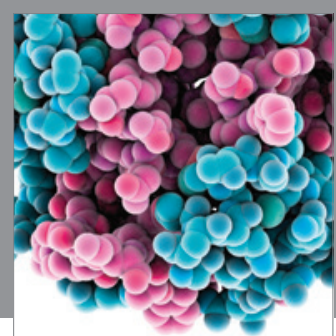

Journal of
Diabetes Research

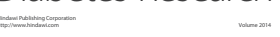

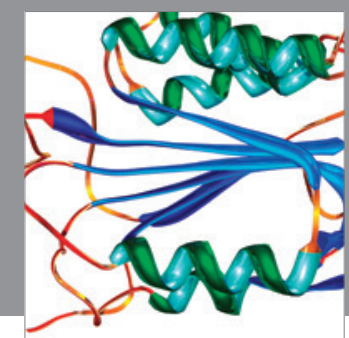

Disease Markers
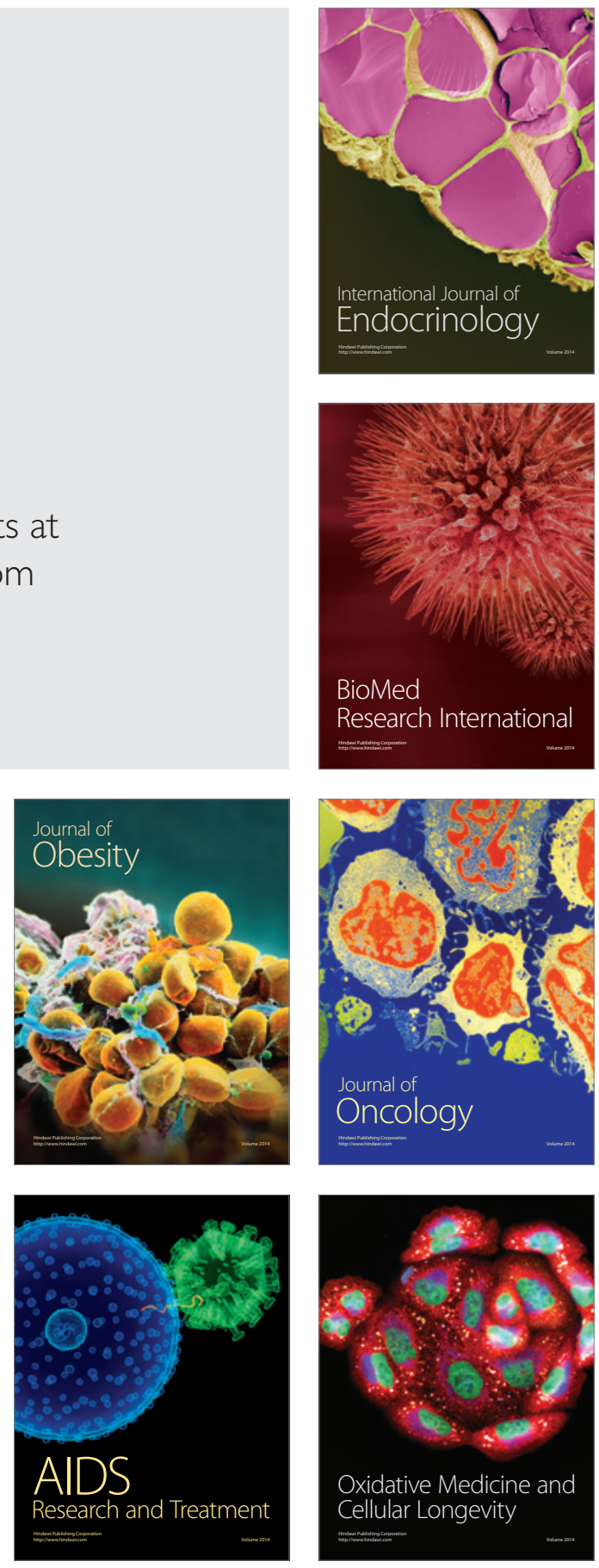